Revue d'histoire de l'Amérique française

\title{
La mine qui " commence à grignoter le village » : expansion minière territoriale à Asbestos
}

\section{Jessica van Horssen}

Volume 68, numéro 3-4, hiver-printemps 2015

URI : https://id.erudit.org/iderudit/1033639ar

DOI : https://doi.org/10.7202/1033639ar

Aller au sommaire du numéro

\section{Éditeur(s)}

Institut d'histoire de l'Amérique française

\section{ISSN}

0035-2357 (imprimé)

1492-1383 (numérique)

Découvrir la revue

\section{Citer cet article}

van Horssen, J. (2015). La mine qui " commence à grignoter le village" : expansion minière territoriale à Asbestos. Revue d'histoire de l'Amérique française, 68(3-4), 325-352. https://doi.org/10.7202/1033639ar

\section{Résumé de l'article}

La ville d'Asbestos est le foyer de ce qui était autrefois la plus grande mine d'amiante à ciel ouvert dans le monde, la mine Jeffrey. La majorité de son histoire a été dominée par l'exploitation de la ressource qui lui donna son nom, et aussi la compagnie américaine propriétaire de la mine, la Johns-Manville Company, qui opéra un style agressif de gestion du territoire. En procédant à l'élargissement des limites de la mine dans la communauté afin de répondre à une demande mondiale croissante pour l'amiante, la Johns Manville provoqua des transformations radicales de l'environnement et du paysage local. Face à l'effondrement de l'industrie mondiale de l'amiante, la Johns-Manville intensifia ses plans d'expansion, au grand détriment de la communauté locale. Celle-ci, cependant, n'est pas demeurée muette lors de ces transformations, et cet article examine la façon dont elle a négocié un équilibre entre le progrès et l'épicentre de cette industrie minière. 


\title{
La mine qui “ commence à grignoter le village ${ }^{\mathrm{x}}$ : : expansion minière territoriale à Asbestos
}

\author{
JESSICA VAN HORSSEN \\ Département d'histoire et d'archéologie \\ Université de Chester
}

RÉsumÉ - La ville d'Asbestos est le foyer de ce qui était autrefois la plus grande mine d'amiante à ciel ouvert dans le monde, la mine Jeffrey. La majorité de son histoire a été dominée par l'exploitation de la ressource qui lui donna son nom, et aussi la compagnie américaine propriétaire de la mine, la Johns-Manville Company, qui opéra un style agressif de gestion du territoire. En procédant à l'élargissement des limites de la mine dans la communauté afin de répondre à une demande mondiale croissante pour l'amiante, la Johns Manville provoqua des transformations radicales de l'environnement et du paysage local. Face à l'effondrement de l'industrie mondiale de l'amiante, la Johns-Manville intensifia ses plans d'expansion, au grand détriment de la communauté locale. Celle-ci, cependant, n'est pas demeurée muette lors de ces transformations, et cet article examine la façon dont elle a négocié un équilibre entre le progrès et l'épicentre de cette industrie minière.

ABSTRACT - The town of Asbestos is home to what was once the largest opencast asbestos mine in the world, the Jeffrey Mine. Most of the town's history has been dominated by the resource industry that gave it its name and by the American Johns-Manville Company, which owned the mine and carried out an aggressive style of land management. When it expanded the edges of the mine into the community in order to meet rising global demand for asbestos, the company brought radical changes to the local environment and landscape. As the global asbestos industry began to collapse, Johns-Manville stepped up its expansion plans, much to the detriment of a local community that, however, did not remain voiceless in this process. This paper examines how the community negotiated a balance between progress and place at the epicentre of this mining industry.

1. Le Citoyen, 28 décembre 1974, p. 70. 
L es communautés minières forment des espaces singuliers. Elles sont le foyer de populations entièrement dépendantes d'un environnement naturel qui évolue constamment, au gré de forces indépendantes de tout contrôle local. Si les historiens se sont intéressés aux conditions sociales de ces communautés, ainsi qu'aux conflits ouvriers et aux risques sanitaires, ils ont bien souvent délaissé le rôle fondamental qu'y jouent les enjeux environnementaux et territoriaux ${ }^{2}$. Cet article aborde ces enjeux en étudiant les transformations environnementales de la communauté minière d'Asbestos.

Asbestos abrite la mine Jeffrey, autrefois la plus grande mine d'amiante chrysotile du monde. Durant la majeure partie de son histoire, la mine appartenait et était gérée par la Johns-Manville Company, la plus grande entreprise productrice d'amiante des États-Unis ${ }^{3}$. L'agressive politique d'extraction de cette entreprise s'accompagna de transformations exponentielles et irréversibles du territoire local et de la vie locale. Cet article examine les rapports de force qu'induisait, entre la Johns-Manville et les habitants, la prise de décisions relatives à l'occupation du territoire. Ces rapports tendaient vers un équilibre, mais une hausse de la demande mondiale et les profits qu'elle générait les influençaient fortement. Cet équilibre était également fonction des représentations que les populations locales se faisaient du minerai et de la mine, entre autres lorsque leur santé s'en trouvait menacée. En analysant les phases successives d'agrandissement de la mine de 1918 à 1983, cet article illustre les modes de fonctionnement des sociétés multinationales au sein des communautés dépendantes des ressources, et les modalités d'action des populations locales pour conserver un certain contrôle sur leur territoire.

L'agressivité qui caractérisait la politique d'extraction et d'occupation du territoire par la Johns-Manville s'explique, entre autres, par la richesse du dépôt d'amiante situé directement sous la ville d'Asbestos. Les gisements traditionnels d'amiante - incluant ceux qui se trouvent ailleurs dans la "ceinture de l'amiante» au Québec comme à Thetford Mines - se pro-

2. Voir notamment Thomas G. Andrews, Killing for Coal: America's Deadliest Labor War (Cambridge et Londres, Harvard University Press, 2008); Karen Buckley, Danger, Death, and Disaster in the Crowsnest Pass Mines, 1902-1928 (Calgary, University of Calgary Press, 2004); Barbara Ellen Smith, Digging Our Own Graves: Coal Miners and the Struggle Over Black Lung Disease (Philadelphie, Temple University Press, 1987), Michael A. Amundson, Yellowcake Towns: Uranium Mining Communities in the American West (Boulder, University Press of Colorado, 2002); Larry Lankton, Cradle to Grave: Life, Work, and Death at the Lake Superior Copper Mines (Oxford, Oxford University Press, 1991) et Anthony F.C. Wallace, St. Clair: A Nineteenth-Century Coal Town's Experience with a Disaster-Prone Industry, $3^{\mathrm{e}}$ éd. (New York, Alfred A. Knopf, 1987).

3. Johns-Manville fut propriétaire de la mine Jeffrey de 1918 à 1983. 
filent horizontalement à travers le roc environnant. Y accéder requiert plusieurs mines qui sont souvent détenues par de nombreuses entreprises distinctes. À Asbestos, le gisement est circulaire et il contient peu de débris de roche, ce qui signifie qu'une seule entreprise peut l'exploiter et en récolter seule les bénéfices. Au Québec, comme les droits miniers sont distincts des droits superficiaires, l'entreprise doit s'entendre avec la population locale et les propriétaires fonciers pour accéder à la richesse minérale présente dans le sous-sol ${ }^{4}$. Lorsque la Johns-Manville fit l'acquisition de la mine Jeffrey en 1918, elle établit à Asbestos une communauté dépendante des ressources auprès d'une seule industrie et d'une seule entreprise. La population locale, qui avait traversé l'emballement et l'effondrement de cette industrie pendant quarante ans, réserva initialement un accueil favorable au nouveau propriétaire de la mine.

La Johns-Manville transforma radicalement le territoire et la relation que la communauté entretenait avec celui-ci. L'entreprise et la population d'Asbestos maintenaient un équilibre entre un espace de vie et un espace de travail que la mine Jeffrey délimitait et définissait. Asbestos était littéralement construite au sommet d'un dépôt massif d'amiante, et la fosse constituait un élément central de la vie quotidienne, à proximité de laquelle les habitants souhaitaient vivre. Cette proximité entre le sol et le travail était d'autant plus souhaitable et nécessaire que le gisement était riche et que la terre potentiellement cultivable était éloignée de la mine. D’ailleurs, la mine dont la Johns-Manville fit l'acquisition en 1918 ne laissait entrevoir qu'une maigre portion du gisement d'amiante, et son étendue demeura méconnue durant des décennies. Au cours de la période à l'étude, les habitants comprirent progressivement qu'au fur et à mesure que la Johns-Manville faisait de la mine Jeffrey l'une des mines d'amiante les plus prospères du monde, une partie de leur souveraineté se dissipait.

La Johns-Manville possédait d'autres mines au Canada et aux États-Unis, mais la mine Jeffrey constitua sa principale réserve de matière première, ainsi que sa principale source d'approvisionnement pour ses usines de transformation et ses clients. La situation était idéale pour le gouvernement québécois qui était déterminé à mettre les ressources naturelles de la Province entre les mains d'hommes d'affaires américains afin de garantir le succès industriel du Québec ${ }^{5}$. Cet article s’appuie sur des archives gou-

4. Gouvernement du Québec, Mining Act, chapitre M-13.1 www2.publicationsduquebec.gouv.qc.ca/ dynamicSearch $/$ telecharge.php?type =2\&file=/M_13_1/M13_1_A.html [consulté le 22 juin 2015].

5. Voir notamment Serge Courville, Le Québec: Genèses et mutations du territoire (Québec, Les Presses de l’Université Laval, 2000); Paul-André Linteau, René Durocher et Jean-Claude Robert, Histoire du Québec 
vernementales afin d'analyser l'impact économique qu'eut l'industrie de l'amiante au Québec, sur des archives d'entreprises afin d'éclairer l'histoire voilée de la santé environnementale à Asbestos et sur des archives locales afin de décrypter ce qui se tramait en ces temps de radicales transformations paysagères. Car si la recherche de profits constitua un élément clé de ces transformations, il demeure que c'est la communauté d'Asbestos qui en était l'épicentre, notamment quant aux conséquences de l'agrandissement de la mine. Après tout, cette communauté choisit de vivre au côté de la plus grande mine à ciel ouvert dans le monde, et son histoire nous renseigne sur la façon dont les travailleurs et leurs familles firent face à des conditions aussi dramatiques. L'attrait qu'Asbestos exerçait sur la Johns-Manville découlait en partie du fait que les membres de la communauté dépendaient de l'industrie ${ }^{6}$. Tout, et tous, tournaient autour de la mine.

\section{DE PETITS SACRIFICES, I918-1923}

À la fin de la Première Guerre mondiale, la province de Québec produisait $80 \%$ de l'offre mondiale d'amiante ${ }^{7}$, et les Américains avaient le plus parfait contrôle de l'industrie. Pourtant, le Canadian Mining Journal écrivait que

le futur de l'amiante commence tout juste, [et] la diversité de ses emplois est immense. [Aussi] le Canada, occupant une position si dominante sur le marché, espère fort probablement avec impatience non seulement une production accrue de matière première, mais aussi la multiplication des industries engagées dans la production et la commercialisation de l'amiante sous forme finie ${ }^{8}$.

À l'origine de cette hausse de la demande se trouvaient la reconstruction européenne de l'entre-deux-guerres mais aussi, et surtout, l'industrie automobile américaine et ses garnitures de frein amiantées.

La Johns-Manville transforma rapidement la fosse à ciel ouvert en une gigantesque infrastructure «établie sous forme de larges gradins, [avec] la roche chargée dans des voitures de gabarit standard par des pelles à

Contemporain: De la Confédération à la crise (1867-1929) (Montréal, Les Éditions du Boréal Express, 1979); Thomas F. McIlwraith et Edward K. Muller, dir., North America: The Historical Geography of a Changing Continent (Lanham, Rowman \& Littlefield Publishers, 2001).

6. Centre de ressources pour l'étude des Cantons de l'Est, Fonds W. G. Clarke.

7. A. Leonard Summers, Asbestos and the Asbestos Industry: The World's Most Wonderful Mineral and Other Fireproof Materials (London, Sir Isaac Pitman and Sons, 1920), 8.

8. Canadian Mining Journal, 40 (24 décembre 1919): 966. Nous traduisons. 
vapeur puis tractée en train jusqu'à l'atelier de concassage par des locomotives à vapeur, avec un gradient maximal de $3 \%{ }^{9}$ ». Puis, en 1922, la Johns-Manville s'efforça d'optimiser ce système en "déménageant» la route principale. Non seulement la route Asbestos-Danville longeait la mine Jeffrey, mais en raison de sa proximité avec la fosse, elle était également le lieu où les premiers habitants avaient choisi de s'installer. Le phénomène était caractéristique des communautés de la ceinture de l'amiante. Ainsi, à Thetford Mines, les travailleurs étaient encouragés à vivre à proximité des mines. Dès lors qu'elles avaient besoin d'étendre leurs activités, les entreprises responsables pouvaient déplacer les travailleurs et leurs maisons ${ }^{10}$. Le conseil municipal de la Ville d'Asbestos approuva la proposition de la Johns-Manville: trop proche de la mine, non seulement la route était dangereuse, mais elle devenait un obstacle au progrès industriel «si essentiel à la vie et l'intérêt de la communauté locale ${ }^{11}$ ». Malgré les perturbations générées par le déplacement de la route, seules huit personnes à Asbestos - toutes des hommes d'affaires sans attachement direct à la mine - protestèrent ${ }^{12}$. La mine Jeffrey était d'une importance cruciale pour la majeure partie de la population. Tous les autres usages éventuels du territoire étaient secondaires.

\section{UN SUCCÈS HISTORIQUE, I924- 1938}

Au milieu des années 1920, une industrie automobile en pleine ascension consommait sous une dizaine de formes possibles plus de $50 \%$ des produits manufacturés contenant de l'amiante ${ }^{13}$. Au Québec, l'extraction de 225140 tonnes métriques d'amiante en 1925 (par rapport à une production mondiale d'environ de 312000 tonnes métriques) rapportait au gouvernement provincial $336250 \$$ de recettes fiscales, auxquelles la mine Jeffrey contribuait largement ${ }^{14}$. Enregistrant des profits industriels records, et voyant la population d'Asbestos s'élever à 3602 habitants en 1926, le

9. "Modernization of the Asbestos Mines", Canadian Mining Journal, 42 (5 août 1921): 618. Nous traduisons.

10. François Cinq-Mars et Romain Dubé, Thetford Mines à ciel ouvert: histoire d'une ville minière (Thetford Mines, La Ville de Thetford Mines, 1994), 185. Nous traduisons.

11. Ville d'Asbestos, Procès-verbal, 13 novembre 1923, p. 447.

12. Réjean Lampron, Marc Cantin et Élise Grimard, Asbestos: filons d'histoire, 1899-1999 (Asbestos, Centenaire de La Ville d'Asbestos, 1999), 117.

13. Canadian Mining Journal, 45, 1 (8 avril 1924): 374.

14. Les données relatives au Québec proviennent des rapports Opérations minières (1898-1936) et Industrie minière de la Province de Québec (1937-2000) (www.mern.gouv.qc.ca/mines/desminesetdeshommes/index.jsp\#annexe), compilées par Marc Vallières. J’ai converti la tonne américaine indiquée sur la liste en tonne métrique afin de comparer plus aisément les données avec celles mondiales, fournies par le United States Geological Survey, Circular 1298, Robert L. Virta, «Worldwide Asbestos Supply and 
conseil municipal écrivit en 1927 au gouvernement de la province afin de demander la permission d'agrandir les frontières de la ville; rapidement, Asbestos s'étendait sur 800 acres. La Johns-Manville demanda immédiatement à la ville l'octroi de 55 acres de ces nouvelles terres, en échange du maintien de son approvisionnement en électricité ${ }^{15}$.

La Johns-Manville justifia sa requête en expliquant que la localité se trouvait sur la route nécessaire à l'agrandissement de la mine Jeffrey. Le prix mondial de l'amiante était en hausse, et le Canada participant alors à $85 \%$ de la production mondiale de la fibre, l'entreprise avait là l'occasion parfaite d'accroître sa production. Afin d'endiguer un ressentiment potentiel à l'égard de cet agrandissement, lequel allait de nouveau contraindre les citoyens à s'éloigner de la mine qui empiétait encore davantage sur les quartiers existants, la Johns-Manville promit l'aménagement de quatre nouvelles routes et sur celles-ci, la construction d'habitations locatives pour ses employés et leurs familles qu'elle doterait d'équipements modernes tels que l'eau courante et l'électricité, ainsi qu'un réseau d'éclairage $^{16}$. Au fur et à mesure que l'entreprise remodelait leur environnement, les habitants d'Asbestos lui devenaient redevables et la grande majorité d'entre eux soutenait également les travaux d'agrandissement de la mine.

L'agrandissement de la mine en 1928 fut le premier «à grignoter le village $^{17}$ ». Si cette description semblait conférer à la mine Jeffrey une certaine personnalité et une capacité d'action, la population locale ne voyait pas forcément d'un mauvais œil ce "grignotage»: la mine était ce qui importait. À Thetford Mines, les habitants ne connurent pas de tel empiètement sur leur milieu de vie avant les années 1950, lorsque les mines King, Bell et Johnson s'étendirent au cœur de la communauté ${ }^{18}$. Les différents types de gisements expliquent cette différence : tandis qu'à Thetford, l'expansion minière pouvait se faire de façon linéaire en s'éloignant de la ville, à Asbestos, le dépôt circulaire était situé immédiatement sous la ville. Beaucoup de résidents furent mécontents de la destruction du centre-ville originel. Cependant, la fin des années 1920 constituait une période exaltante pour Asbestos, et la région était internationalement connue comme «le territoire producteur d'amiante le plus important du monde. L'amiante extraite ici [était] une référence pour toute l'indus-

Consumption Trends from 1900 through 2003 ", 2006 (http:/ / pubs.usgs.gov/circ/2006/1298/c1298.pdf [consulté le 22 octobre 2014]) p. 32-34.

15. Ville d'Asbestos, Procès-verbal, 27 avril 1927, p. 137.

16. R. Lampron et al., Asbestos..., op.cit., 140.

17. Le Citoyen, 28 décembre 1974, p. 70.

18. R. Cinq-Mars et R. Dubé, Thetford Mines à ciel ouvert..., op.cit., 385. 
trie $^{19} \ldots$... Les communautés minières du Québec fournissaient $63 \%$ de l'offre mondiale d'amiante en 1928, et elles rapportaient $522629 \$$ de recettes fiscales au gouvernement provincial ${ }^{20}$. Un territoire aussi réputé ne serait jamais assez exploité.

Cependant, le prix mondial de l'amiante chuta fortement au début de la Grande Dépression, et la Johns-Manville réduisit de façon draconienne la main-d'œuvre employée à la mine Jeffrey ${ }^{21}$. Parce qu'elle était inextricablement liée aux industries de l'automobile et de la construction alors en plein effondrement, la Johns-Manville subissait la Dépression de plein fouet. Du jour au lendemain, le sous-sol d'Asbestos avait perdu sa valeur.

Pour les habitants d'Asbestos qui avaient volontairement sacrifié leurs terrains et leurs habitations pour l'agrandissement de la mine Jeffrey en 1928, la Grande Dépression et la perte d'emploi qui s'ensuivit ne purent les convaincre qu'ils avaient pris la bonne décision. Malgré la surproduction mondiale, la hausse de la production de la fibre en Union soviétique et les nouveaux sondages géologiques destinés à localiser d'autres gisements au Québec, la Johns-Manville poursuivit ses projets. L'agrandissement de la mine Jeffrey sur 55 acres détruisit ce que les gens du cru appelaient le "centre névralgique» de la ville, incluant Le Carré, magasin qu'avait ouvert en 1890 le premier maire d'Asbestos, Henri Roux $^{22}$. Pour contrebalancer ces pertes, la Johns-Manville céda à la ville une quantité équivalente de terrains où construire un nouveau centre-ville commercial. L'entreprise reçut l'aval du conseil pour aller de l'avant, ce qui n'empêcha pas les commerçants locaux de protester.

Défendant l'agrandissement pour les emplois qui en découleraient, le conseil municipal soutint la Johns-Manville, qui «apport[ait] la presque totalité des revenus d'Asbestos ${ }^{23}$ ». Médecin à Asbestos, le Dr Elzéar Émard protesta officiellement en 1930 contre la destruction du magasin Le Carré, et déclara que le conseil défendait non pas l'intérêt de la communauté, mais celui de la Johns-Manville ${ }^{24}$. Émard avait beau exercer à Asbestos, il ignorait le résultat des examens médicaux des employés de la mine Jeffrey. En effet, la Johns-Manville possédait et gérait l'hôpital d'Asbestos où les médecins

19. Asbestos: Its Sources, Extraction, Preparation, Manufacture, and Uses in Industry and Engineering (Berlin, Becker and Haag, 1928), 17. Nous traduisons.

20. M. Vallières, www.mern.gouv.qc.ca/mines/desminesetdeshommes/index.jsp\#annexe, [consulté le 12 janvier 2015].

21. Elizabeth W. Gillies, The Asbestos Industry Since 1929 with Special Reference to Canada, mémoire de maîtrise (économie), McGill University, 1941, p. 40.

22. Le Citoyen, 28 décembre 1974, p. 42.

23. L'Asbestos, 26 août 1949 , p. 1.

24. Le Citoyen, 28 décembre 1974, p. 70. 
rattachés à l'entreprise procédaient à l'examen de chaque employé, sans pour autant les informer des résultats de leurs examens. D’ailleurs, pour le docteur de la Johns-Manville à Asbestos, Kenneth Smith, il importait que les médecins qui ne travaillaient pas pour l'industrie de l'amiante ne puissent avoir accès aux mineurs. Pour Smith, seule l'industrie de l'amiante devait être en mesure de faire un suivi des maladies professionnelles, sans qu'elle n'ait à alerter les travailleurs, ni le grand public, des risques encourus ${ }^{25}$. C'est uniquement en tant que citoyen qu'Émard avait formulé cette observation et, bien que le conseil lui demanda de se rétracter, son accusation demeura valide. La communauté souhaitait encourager les succès de la JohnsManville, mais l'entreprise ne contrôlait par les affaires de la ville et une certaine distance entre les deux devait être établie. Pour éviter toute accusation de partialité, le maire fit appel au gouvernement provincial pour statuer sur l'agrandissement de la mine.

Réticent à freiner tout projet apte à fournir du travail, le gouvernement du Québec prit le parti de la Johns-Manville en 1931, et le premier projet de loi d'expropriation à Asbestos fut mis en œuvre. Puisque la vie même de la ville dépendait de la mine et que la Johns-Manville était responsable de cette mine, l'entreprise était finalement à la tête d'Asbestos, et l'expropriation consolida cet état de fait. Sous le règne de la Johns-Manville, la ville était dépossédée de son passé. Plutôt, elle disposait d'un futur que seules garantissaient des transformations territoriales sans limite. Malgré le succès annoncé, la Johns-Manville fut contrainte de fermer complètement la mine Jeffrey entre mai 1932 et avril 1933, les effets de la Dépression se faisant ressentir sur le marché mondial de l'amiante.

Durant la Dépression, la Johns-Manville développa de nouveaux marchés pour l'isolation des chaudières et des tuyaux de magnésium afin de pallier la chute de la demande de l'industrie automobile ${ }^{26}$. Grâce à ces innovations, mais aussi grâce au regain soudain du secteur automobile à la fin de l'année 1933, l'industrie de l'amiante connut une hausse annuelle de production de $29 \%$ et une augmentation de sa valeur monétaire de $71 \%{ }^{27}$. La mine Jeffrey rouvrit ses portes, et si le niveau de production antérieure à la Dépression ne fut retrouvé qu'après la Seconde Guerre mondiale, les employés travaillaient en moyenne 58 heures par semaine

25. Kenneth Smith, C-JM Asbestos, à Ivan Sabourin, JM, 13 mars 1947. "Asbestos Chronology», Asbestos Claims Research Facility, Aurora, Colorado, p. 40.

26. Jock McCulloch et Geoffrey Tweedale, Defending the Indefensible: The Global Asbestos Industry and its Fight for Survival (Oxford, Oxford University Press, 2008), 26.

27. Oliver Bowles et B. H. Stoddard, "Asbestos», dans O. E., Kiessling, dir., Minerals Yearbook 1934 (Washington, United States Government Printing Office, 1934), 1014. 
en 1935. Les autres communautés minières de la région peinaient quant à elles à satisfaire la hausse de la demande, car l'espace physique manquait pour étendre leurs activités. Ce n'était pas le cas à Asbestos où en matière d'occupation du territoire, l'industrie l'emportait sur la communauté. D'ailleurs, en 1938, la Johns-Manville proposa de nouveau au conseil municipal d'agrandir la mine Jeffrey.

\section{QUAND RÉVOLUTION INDUSTRIELLE ET PHÉNOMÈNE NATUREL SE RENCONTRENT, 1938-1949}

Les années qui suivirent la Dépression apprirent à la population d'Asbestos que si elle accordait sa confiance à la mine Jeffrey et à la Johns-Manville, elle prospérerait. Le conseil municipal approuva sans hésitation la requête de l'entreprise relative à l'octroi de quatorze parcelles non exploitées ${ }^{28}$. L'agrandissement n'affectait pas directement les foyers et les commerces de la ville, et il n'était pas non plus aussi controversé que le premier grand élargissement de la mine.

Aux yeux de la population locale, il allait de soi que la fosse, qui ressemblait à un cône inversé et escarpé avec, à son extrémité, un accès difficile à la fibre, avait clairement atteint ses limites ${ }^{29}$. Afin d'éviter les éboulis de falaise, les travailleurs devaient d'abord élargir la fosse avant de creuser plus profondément. Pour maintenir à la fois la stabilité structurelle du sol et la stabilité financière de la ville, la mine Jeffrey devait être agrandie.

En 1939, alors que le marché de l'amiante se mettait à croître de façon exponentielle après le déclenchement de la Seconde Guerre mondiale, la mine Jeffrey était déjà connue pour être la mine d'amiante la plus grande du monde ${ }^{30}$. Pour le minerai comme pour Asbestos, trente années de prospérité débutèrent. En 1941, stimulées par les industries de guerre et la sortie temporaire de la fibre soviétique du marché, les ventes de la Johns-Manville augmentèrent de $50 \%{ }^{31}$. À la fin de l'année, la JohnsManville s'enquit auprès du conseil municipal de la possibilité de poursuivre l'agrandissement de la mine par l'acquisition de terrains situés à proximité du cœur de la ville. Considérant que l'entreprise ne pouvait nuire en cette période de prospérité ${ }^{32}$, le conseil donna suite à cette

28. Ville d'Asbestos, Procès-verbal, 30 septembre 1938, p. 158.

29. W. Gillies Ross, "Encroachment of the Jeffrey Mine on the Town of Asbestos, Quebec», Geographical Review, 57, 4 (1967): 529.

30. R. C. Rowe, «Mining and Milling Operations of the Canadian Johns-Manville Company Ltd. at Asbestos, PQ", Canadian Mining Journal (avril 1939): 185.

31. J. McCulloch et G. Tweedale, Defending the Indefensible..., op. cit., 28.

32. Ville d'Asbestos, Procès-verbal, 6 mai 1942, p. 495. 
demande au printemps 1942. En retour, la Johns-Manville donna à la ville $5000 \$$ pour une route de moins de 500 pieds. Propriétaire à Asbestos, Joseph A. Lambert demanda immédiatement au conseil l'autorisation de déplacer sa maison, à ses frais, à l'écart de la route. Entre juin 1942 et février 1943, quatre autres résidents demandèrent au conseil la permission de déplacer leurs habitations pour les mettre à l'écart de la fosse grandissante $^{33}$. Plutôt que de tenter de limiter l'expansion de la mine, ou d'attaquer l'entreprise en justice pour les coûts associés au déplacement de leurs maisons, les résidents libérèrent tout simplement le passage.

En 1943, la mine Jeffrey s'étendait sur 115 acres de terre, et les travailleurs extrayaient quotidiennement 6000 tonnes de roches et de minerai. Pareille activité contribua de façon significative à la production des 384495 tonnes métriques - 50\% de l'offre mondiale - qui rapportèrent cette année-là au Québec quelque $2046945 \$$ de recettes fiscales ${ }^{34}$. La Johns-Manville n'était pas en mesure d'étendre la mine assez rapidement pour répondre à la demande industrielle en amiante, surtout après l'entrée en guerre des États-Unis en 1941. La mine Jeffrey se retrouvait si proche de la zone résidentielle d'Asbestos que les eaux usées provenant des maisons en bordure de la mine commençaient à se déverser dans la fosse et à contaminer la fibre ${ }^{35}$. En réaction à ce problème inédit, au début de l'année 1944, le président de la Johns-Manville G. K. Foster informa le conseil municipal que l'extraction se ferait dorénavant sous terre.

Si l'extraction minière souterraine n'était pas particulièrement usitée pour l'amiante - nulle part ailleurs dans la région on y avait recours - elle permit à la Johns-Manville d'élargir son champ d'action et d'accroître rapidement la production sans pour autant empiéter sur la propriété des habitants, les puits et les galeries nécessitant nettement moins d'espace. Les deux premiers puits étaient larges et hauts de 10 pieds, et profond de 750 pieds environ. Chacun d'entre eux était équipé d'une courroie transporteuse d'une hauteur de 25 pieds convoyant le minerai hors du sol et vers les ateliers, de façon à éviter la projection de roches sur les quartiers voisins $^{36}$. Le conseil municipal approuva la construction de ces puits et en retour, la Johns-Manville offrit une partie de ses terrains afin d'y prolonger l'une des routes de la ville. Plutôt que de mécontenter la population locale

33. Ville d'Asbestos, Procès-verbal, 22 juin 1942, p. 3 et 5, et 8 février 1943, p. 36.

34. M. Vallières, www.mern.gouv.qc.ca/mines/desminesetdeshommes/index.jsp\#annexe [consulté le 12 janvier 2015].

35. Ville d'Asbestos, Procès-verbal, 16 juin 1943, p. 50 et 7 juillet 1943, p. 52.

36. Ville d'Asbestos, Procès-verbal, 9 août 1944, p. 8. 
en poursuivant l'agrandissement, la Johns-Manville choisit la méthode d'extraction la plus rapide, la moins chère et la moins invasive. La décision de l'entreprise de recourir à un autre mode d'extraction indique qu'un mécontentement se généralisait chez les habitants d'Asbestos face à l'agrandissement permanent de la fosse et l'expropriation répétée des terres. Associée à l'exploitation à ciel ouvert, l'extraction souterraine permettait à la Johns-Manville d'accroître la production tout en maintenant de bonnes relations avec la communauté.

En revanche, la Johns-Manville ne parvenait pas à apaiser les commerçants locaux. Entre l'entreprise et les petits commerçants d'Asbestos, les tensions étaient constantes. Employés à la mine Jeffrey, la majorité des habitants prenaient généralement parti pour l'entreprise sur les enjeux territoriaux, tandis que les propriétaires fonciers représentaient une minorité bruyante opposée à la Johns-Manville. Lorsque la mine "grignota " Le Carré en 1930, le centre-ville commercial et originel disparut et les commerçants cherchèrent à créer un nouveau quartier, rue Bourbeau. C'est pourtant à cet endroit que la Johns-Manville choisit de construire les puits d'extraction. En novembre 1946, lors d'une séance du conseil municipal, l'entreprise demanda l'autorisation de fermer une grande partie de la rue Bourbeau et de la rue Saint-Georges qui lui était adjacente, pour introduire ses nouvelles techniques d'extraction. La Johns-Manville eut beau proposer le prolongement d'une autre route et offrir toute l'aide nécessaire pour "défendre les intérêts de la Ville d'Asbestos», les commerçants de la rue Bourbeau demeuraient insatisfaits ${ }^{37}$. Durant la même séance, la Ligue des Propriétaires d'Asbestos présenta une déclaration dans laquelle elle implorait le conseil de prendre des mesures à l'endroit de la Johns-Manville pour contrecarrer toute activité menaçant la valeur de leurs propriétés et de leurs commerces ${ }^{38}$. Il s'agissait d'un sérieux enjeu conflictuel touchant l'occupation du territoire. Tandis que les hommes d'affaires locaux cherchaient avant tout à éviter toute perte de revenu et de valeur mobilière, la stratégie foncière de la Johns-Manville offrait à la communauté stabilité et profits. Le maire Albert Goudreau demanda alors au conseil de prendre une décision ferme en tenant compte de l'avenir économique de la communauté.

Le conseil approuva la demande de la Johns-Manville de fermer la route en $1947^{39}$ et Goudreau invita les commerçants à déposer leurs plaintes

37. Ville d'Asbestos, Procès-verbal, 6 novembre 1946, p. 199.

38. Ville d'Asbestos, Procès-verbal, 6 novembre 1946, p. 199.

39. Ville d'Asbestos, Procès-verbal, «Règlement n 240 », 1947, p. 241. 
auprès de l'entreprise. Le développement territorial et économique à Asbestos découlait d'abord des priorités de la Johns-Manville, et ce, d'autant plus que la demande pour le minerai ne cessait de croître depuis la Seconde Guerre mondiale : l'industrie québécoise exportait 10785189 tonnes de fibre en 1947, pour une valeur de $438356805 \$ \$^{40}$. Grâce à la combinaison d'une fosse à ciel ouvert, de puits d'extraction et d'un nouveau système de foudroyage par blocs, les niveaux de production de la mine Jeffrey surpassaient, de loin, ceux de ses compétiteurs régionaux. Le foudroyage par blocs est une forme d'extraction souterraine tout particulièrement adaptée à un terrain sur lequel se trouve une fosse à ciel ouvert en fonction. Les gradins en spirales qui menaient au sommet de la mine Jeffrey, et qui permettaient aux locomotives et aux camions d'apporter la fibre jusqu’à la surface, étaient creusés et minés depuis l’intérieur. Les hommes travaillaient sur d'immenses racleurs mécaniques à doubletambour pour remonter la roche depuis la mine jusqu'à la surface, chargeant dans des wagons le minerai à envoyer à l'usine. Le foudroyage par blocs industrialisa encore davantage le territoire, sans que cela ne soit visible depuis la surface, et fragilisa encore davantage la structure en gradins de la mine Jeffrey.

En 1948, la mine s'étendait sur 3200 pieds de long et 2800 pieds de large, atteignant la limite des propriétés détenues par la Johns-Manville. Le foudroyage par bloc permit à l'entreprise de poursuivre son activité sans acheter davantage de terrains à la ville, et constitua ainsi une façon économiquement et politiquement efficace pour extraire des quantités considérables de roche, entre 19000 et 22760 tonnes par jour ${ }^{41}$. La transition vers l'extraction souterraine signale une nouvelle fois la réticence de la Johns-Manville à exproprier plus de terrains pour agrandir la mine Jeffrey, probablement à cause des relations tendues entre l'entreprise et la communauté. En effet, les travailleurs n'appréciaient guère d'être remplacés par de nouvelles technologies, même si, par ailleurs, les habitants d'Asbestos s'étaient accoutumés au bruit et à la poussière émanant constamment de la mine Jeffrey.

Face à la hausse des activités d'extraction, le conseil municipal anticipa une hausse des embauches à la Johns-Manville, ce qui signifiait que la communauté aurait besoin de plus de maisons. Conséquemment, le conseil acheta des terrains pour l'expansion tout au long de l'année 1948

40. H. R. Rice, "The Asbestos Industry in Quebec», Canadian Mining Journal, 59, 11 (octobre 1948): 148.

41. Ibid., 159. 
pour répondre à cette hausse prévue de la population. En mars et avril 1948, la ville acheta à la Johns-Manville un terrain de 16605 pieds et elle annexa 8 autres parcelles du comté en prévision d'une expansion future ${ }^{42}$. Ces opérations se déroulèrent aisément mais, lorsqu'il fut question d'acheter des terrains aux propriétaires locaux, la ville eut plus de mal: trois citoyens refusèrent de vendre leur terre ${ }^{43}$. Les habitants contrôlaient davantage le mode d'occupation du territoire à Asbestos. Le Conseil avait beaucoup appris de la Johns-Manville et elle menaça les propriétaires récalcitrants de son intention de les exproprier. Bien que l'économie locale demeurait complètement dépendante de la mine Jeffrey, la population locale cessait d'entretenir avec la Johns-Manville le même type de rapports que ceux précédemment établis.

\section{LA LUTTE POUR LA PROPRIÉTÉ, I949- 1959}

La mine Jeffrey avait déjà été le site de conflits de travail, mais au début de l'année 1949, une grève prolongée, âpre et brutale frappa la communauté et avec elle, pendant 5 mois, toute l'industrie québécoise de l'amiante. Un des principaux enjeux de la grève résidait dans les problèmes de santé qui affligeaient les travailleurs. Le vice-président de la JohnsManville, Vandiver Brown, écrivit en octobre 1948 à d'autres dirigeants industriels qu'en raison d'une prise de conscience croissante des dangers auxquels l'amiante expose la santé des hommes, un "problème est en train de naître chez les mineurs du Québec ${ }^{44}$ ». Néanmoins, le territoire était, au même titre que la santé, au cœur des préoccupations des travailleurs, car dans les deux cas, la qualité de vie de la communauté minière était en jeu. Dans une lettre ouverte à ses investisseurs, le président de la JohnsManville Lewis H. Brown écrivit que l'enjeu de la grève de 1949 était "l'insistance des dirigeants syndicaux à obtenir un certain contrôle sur la gestion de l'entreprise. C'est la doctrine révolutionnaire selon laquelle le droit des propriétaires jusqu'ici incontesté à définir les modes de gestion de la propriété doit être soumis au veto de la direction syndicale ${ }^{45}$. " Le contrôle local du mode d'occupation du territoire était ce qui, en fait, animait les travailleurs de la mine Jeffrey, exaspérés qu'ils étaient de voir la mine continuellement empiéter sur leur milieu de vie.

42. Ville d'Asbestos, Procès-verbal, mars 1948, p. 280 et 26 avril 1948, p. 282.

43. Ville d'Asbestos, Procès-verbal, 7 juillet 1948, p. 16 et mars 1948, p. 280.

44. Vandiver Brown, VP JM, à Ernest Muehleck (Keasby \& Mattison) et Rohrbach, RaybestosManhattan, 22 octobre 1948. Asbestos Claims Research Facility, Aurora, Colorado. Nous traduisons.

45. The Globe and Mail, 23 avril 1949, p. 7. Nous traduisons. 
Lorsque la grève prit fin, la vie dans les communautés minières d'amiante au Québec suivit relativement le même cours qu'auparavant, avec quelques différences significatives. Les habitants, la Johns-Manville et le conseil municipal achetèrent autant de terrains qu'ils le pouvaient. Compte tenu du caractère expansif de la mine Jeffrey, les terrains et leurs propriétaires changeaient continuellement, au fur et à mesure que la fosse grandissait et que les perceptions des gens face à celle-ci évoluaient (figure 1). Les associations locales de constructeurs d'habitations transformaient et le mode de vie et le lieu de vie des travailleurs de la mine Jeffrey. Auparavant, ces derniers louaient leur logement à la Johns-Manville, mais comme on pouvait acquérir ces nouvelles habitations, les travailleurs pouvaient s'affranchir de l'entreprise. Alors que depuis des décennies, il suffisait de déplacer les locataires, agrandir la mine Jeffrey sur des terrains où se trouvaient ces habitations pouvait dorénavant poser problème et compromettre les rapports entre l'entreprise et la communauté. De fait, la propriété privée posa problème.

L’industrialisation du territoire au cours des années 1950 se fit de façon massive, mais subtile. Elle se produisit à travers l'ensemble de la propriété de la Johns-Manville, mais dans des blocs foudroyés et des puits miniers si profonds qu'elle échappait à l'attention des habitants d'Asbestos. Cette subtilité empêcha la communauté de mesurer l'ampleur de la progression des activités d'extraction. Dans la mesure où ils n'avaient pu s'apercevoir que la mine Jeffrey se rapprochait toujours davantage des terres de la communauté, les habitants d'Asbestos furent pris au dépourvu lorsque la Johns-Manville eut besoin d'élargir la fosse.

La Johns-Manville avait considérablement accru la production de l'amiante qui atteignait, en 1954, 735852 tonnes métriques, pour une valeur avoisinant les 80 millions de dollars. Ces chiffres amenèrent le Canadian Mining Journal à déclarer que les avancées technologiques qui permirent à la mine Jeffrey de fournir le tiers de l'offre mondial cette année-là «ferai[en]t de l'exploitation de l'amiante la principale industrie du Québec pour au moins encore un siècle ${ }^{46}$ ». À Asbestos, la prospérité et la stabilité venaient du territoire. Il était inimaginable que quoique ce soit puisse entraver le succès futur.

Anticipant une croissance démographique concomitante à la prospérité de la mine, le conseil municipal acheta les terres agricoles environnantes 


\section{Figure I}

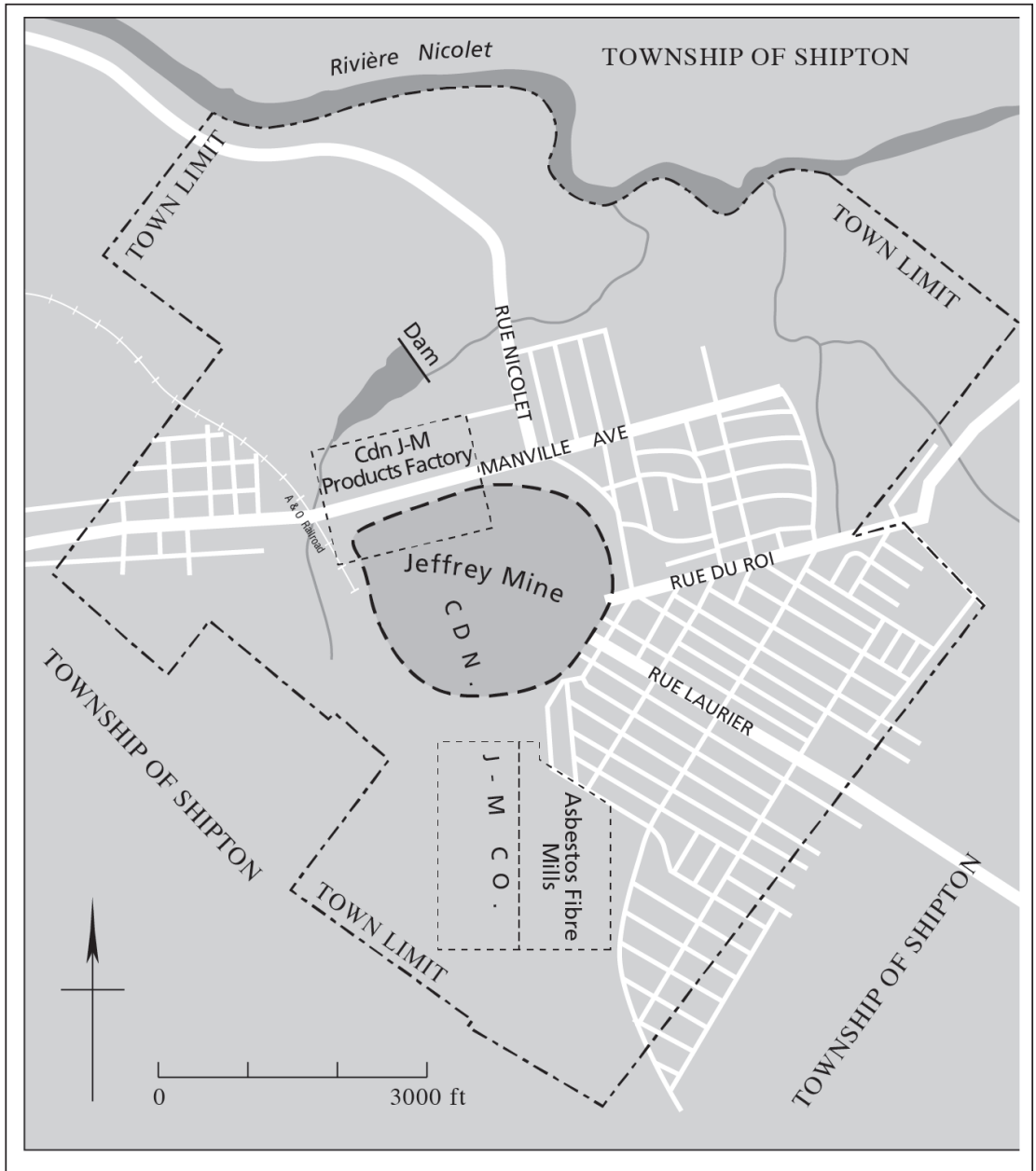

Ce plan d'assurance de la ville d'Asbestos de 1953 nous présente les principales artères évoquées dans cet article, en plus de nous donner une idée de la taille de la mine et de celle de la communauté.

Réalisation: Eric Leinberger, d'après Insurance Plan of the Town of Asbestos, Québec, 1953. Reproduit avec la permission de UBC Press. 
pour repousser les limites de la ville ${ }^{47}$. Les constructeurs locaux bâtirent 124 maisons durant la seule année 1956, ce qui fit d'eux, et non de la Johns-Manville, le principal fournisseur de logements à Asbestos. Si l'accession à la propriété était un signe de prospérité, le rédacteur en chef du journal local s'inquiétait: si ce que prétendait le Canadian Mining Journal s'avérait exact, et que l'industrie continuait de croître durant les cent prochaines années, les propriétaires seraient constamment contraints de sacrifier leur terrain à la mine Jeffrey, jusqu'au jour où ils ne vivraient tout simplement plus à Asbestos. En fait, Asbestos cesserait d'exister en tant que communautét8.

Qualifiée par le Canadian Mining Journal de "géant de l'industrie», parce que la mine Jeffrey fournissait $60 \%$ des exportations canadiennes de fibre, la Johns-Manville engrangea à la fin des années 1950 des profits records, dépassant les 100 millions de dollars en $1957^{49}$. L'entreprise décida de mettre un terme à ses difficiles activités souterraines à Asbestos pour se concentrer uniquement sur la fosse à ciel ouvert. Ses administrateurs y voyaient là une bonne décision efficace. La fosse pouvait être industrialisée bien plus aisément que des puits souterrains, et les nouvelles technologies qui pouvaient y être employées demandaient moins de main-d'œuvre. La hausse des bénéfices et les nouveaux rapports entre l'entreprise et la communauté permettaient aux administrateurs de ne plus se préoccuper du bien-être local. En janvier 1958, la Johns-Manville présenta au conseil municipal son projet d'agrandissement de la mine Jeffrey au cœur de la communauté.

L'agrandissement serait progressif, mais massif. Depuis la réintroduction des méthodes d'exploitation à ciel ouvert, l'extraction se faisait au-delà des limites invisibles des puits. Cela signifiait qu'une grande partie de la zone tampon entre la fosse et la communauté avait déjà été minée, et serait immédiatement consommée. En retour, l'entreprise donnerait à la ville quelques-uns de ses terrains non utilisés au-delà de la fosse. Un autre problème surgissait alors, puisque la rue Saint-Aimé, où se trouvait l'église principale de la ville, serait détruite. Le conseil approuva de nouveau l'agrandissement, qu'il jugeait nécessaire au bien-être de la communauté ${ }^{50}$. L'entreprise fit alors clairement comprendre qu'elle n'avait aucunement l'intention d'employer davantage de travailleurs, et elle mit

47. Ville d'Asbestos, Procès-verbal, 21 juillet 1955, p. 236.

48. Le Citoyen, 26 juillet 1956, p. 2.

49. Canadian Mining Journal, 68, 2 (février 1957), 107 et Le Citoyen, 20 décembre 1957, 1.

50. Ville d'Asbestos, Procès-verbal, 20 mai 1958, p. 174. 
le conseil en garde quant à l'achat de terrains additionnels destinés à accueillir une population grandissante ${ }^{51}$.

Le fait que la Johns-Manville n'eut pas l'intention d'employer davantage de travailleurs après l'agrandissement constitua une rupture radicale dans l'équilibre entreprise-communauté que connaissait Asbestos depuis plusieurs décennies. En licenciant 80 de ses mineurs souterrains en juillet 1958 , et 40 autres en avril $1959^{52}$, l'entreprise illustra le fait que sur le territoire d'Asbestos, seule comptait l'activité minière.

Les habitants étaient inquiets. Pour retrouver un certain contrôle sur le mode d'occupation du territoire, ils votèrent contre l'annexion proposée de terrains additionnels lors d'un référendum, tout en attendant la réaction de la Johns-Manville ${ }^{53}$. L'entreprise orchestra une transformation rapide et radicale du territoire, touchant les populations riveraines des rues Laurier, Panneton, Lafrance, Saint-Jacques, Saint-Dominique, SaintAimé, Legendre, St-George et Amyot. Elle fournit les équipements nécessaires à la construction de nouvelles routes destinées à accueillir les familles déplacées. Malgré cela, forte de son succès économique et de la détermination de ses administrateurs, la Johns-Manville continua de dicter le mode d'occupation du territoire à Asbestos ${ }^{54}$. Les habitants étaient incapables d'enrayer le processus.

\section{«ASBESTOS DOIT PRODUIRE PLUS D'AMIANTE ", | 960 - 197 I}

En 1960, la valeur des expéditions d’amiante au Québec dépassa les 100 millions de dollars. L'industrie rapporta au gouvernement provincial 4691000 \$ de recettes fiscales, et employait environ 6000 travailleurs $^{55}$. À cette époque, la Johns-Manville diversifia sa gamme de produits issus de l'exploitation de l'amiante, comme l'addition de fibres à l'asphalte pour améliorer la durabilité des routes. Anticipant une expansion de ce marché, elle embaucha davantage de personnel. Le phénomène incita le journal local à déclarer qu' "Asbestos $\mathrm{d}[\mathrm{eva}]$ it produire plus d'amiante ${ }^{56}$ ", tandis que la communauté atteignait 10709 membres. La popularité de la mine Jeffrey grandissait au fur et à mesure que de nouveaux usages stimulaient

51. Ville d'Asbestos, Procès-verbal, 27 mai 1958, p. 176.

52. Le Citoyen, 25 juillet 1958, p. 1 et 17 avril 1959, p. 1.

53. Le Citoyen, 15 mai 1959, p. 1.

54. Procès-verbal, Ville d'Asbestos, 19 septembre 1959, p. 48-49.

55. M. Vallières, www.mern.gouv.qc.ca/mines/desminesetdeshommes/index.jsp\#annexe.

56. Le Citoyen, 18 mars 1960, p. 1. 
la demande, et en 1961, l'industrie de l'amiante rapporta 130 millions de dollars au Québec ${ }^{57}$.

$S$ 'ils avaient conscience que la prospérité de la communauté dépendait de l'agrandissement de la mine, les habitants s'indignèrent rapidement devant l'entreprise de destruction inhérente au projet. Propriétaires et commerçants refusèrent de vendre leur propriété et un affrontement autour de l'occupation du territoire débuta. Consciente de l'importance vitale de l'industrie pour l'économie de la Province et forte d'une nouvelle année record en termes de bénéfices, la Johns-Manville sollicita le gouvernement du Québec en 1964. Le ministre des Richesses naturelles, René Lévesque, déclara qu'il adopterait un projet de loi d'expropriation pour l'agrandissement de la mine Jeffrey si la ville y consentait ${ }^{58}$.

Le soutien qu’apporta le ministre Lévesque à la Johns-Manville révéla les limites du pouvoir du gouvernement provincial dans sa capacité de protéger la communauté face à la croissance industrielle. Les citoyens redoutaient l'agrandissement de la mine (figure 2) et la création massive de «terrains morts» qui s'ensuivrait. Le journal local exprima cependant une autre opinion. Il estima que si la Johns-Manville n'était pas autorisée à s'étendre où et lorsque bon lui semblait, l'entreprise quitterait la ville et Asbestos deviendrait une "ville fantôme $e^{59}$ ". Comme leurs moyens de subsistance dépendaient de l'utilisation continue du territoire par la JohnsManville, les habitants autorisèrent l'agrandissement. En 1964, l'entreprise acheta à la communauté l'église de la rue Saint-Aimé pour 1,2 million de dollars ${ }^{60}$. Elle offrit également les terrains nécessaires à la construction de deux nouvelles églises. Les propriétaires de la rue Bourbeau furent moins accommodants. Refusant d'abandonner leurs entreprises à la mine Jeffrey, ils se rendirent à Québec pour s'opposer au projet de loi. Si leurs plaintes furent entendues, le gouvernement du Québec avait néanmoins conscience que seule l'extraction du minerai pouvait garantir la stabilité économique d'Asbestos. Le projet de loi 192 autorisant l'expropriation fut voté en juin 1964.

Le gouvernement exigea de l'entreprise qu'elle attende trois ans pour mettre en œuvre son projet d'agrandissement, et que durant cette période elle négocie des ventes de terrains équitables avec les résidents locaux. Bien que cela semblât raisonnable, les résidents d'Asbestos refusèrent de

57. "Asbestos», Canadian Mining Journal, 83, 2 (février 1962): 117.

58. Le Citoyen, 30 janvier 1964, p. 1.

59. Ibid., p. 1.

60. Le Citoyen, 13 février 1964, p. 1. 


\section{Figure 2}

Les agrandissements passés et prévus de la mine Jeffrey en 1967

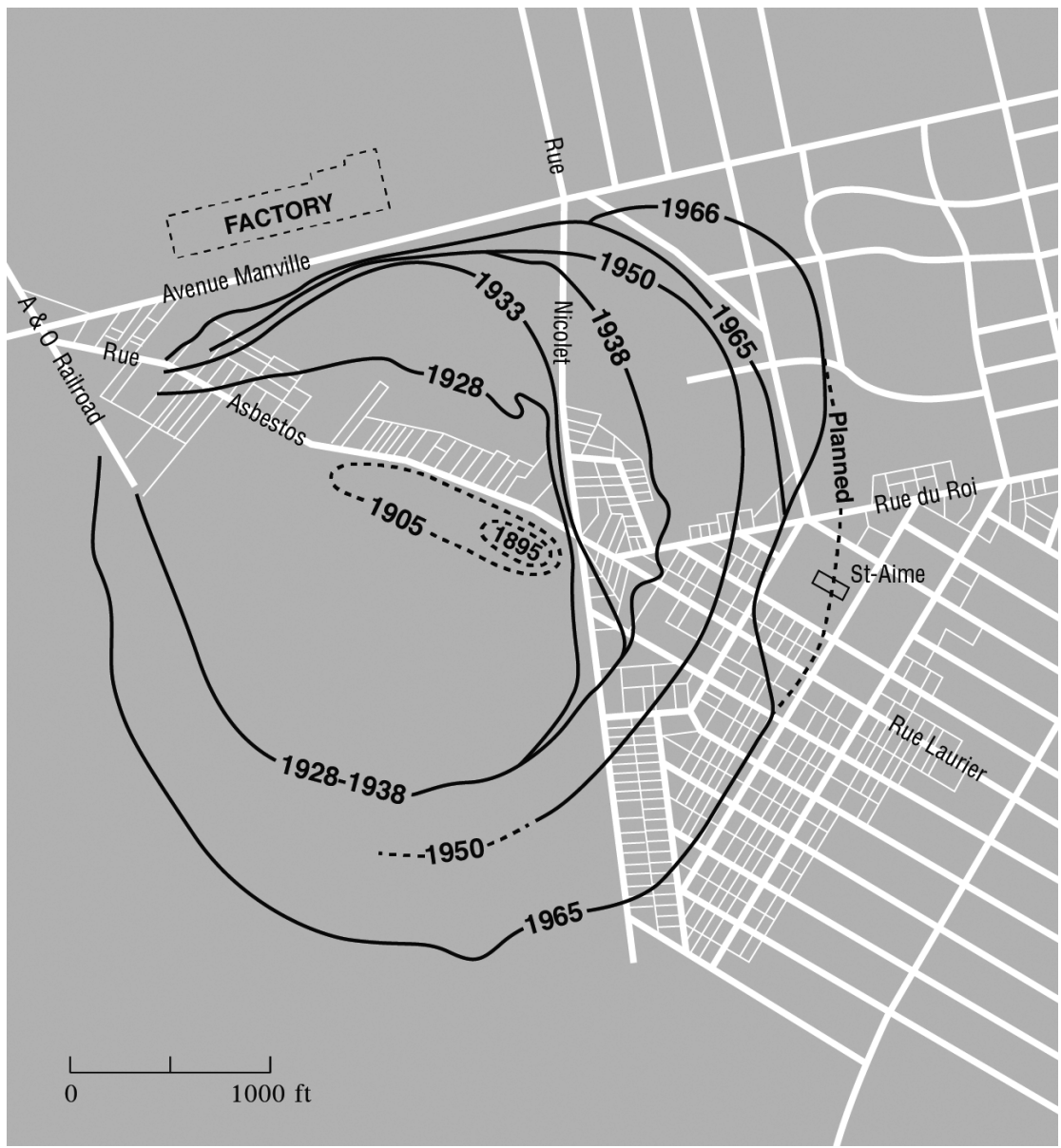

Réalisation: Eric Leinberger, d'après W. Gillies Ross, «Encroachment of the Jeffrey Mine on the Town of Asbestos, Quebec », Geographical Review, 57, 4 (1967): 529. Reproduit avec la permission de UBC Press. 
vendre leurs propriétés. La Johns-Manville en appela du conseil pour qu'il procède à l'achat forcé des terres, pour les lui revendre par la suite ${ }^{61}$. Le rédacteur en chef du journal local somma la population de réfléchir aux bénéfices, et non aux pertes, engendrés par l'agrandissement. Asbestos était née d'une tradition de construction et de reconstruction destinée à satisfaire les besoins de la mine Jeffrey, et était ainsi devenue, à ses yeux, une "ville qui se déplace ${ }^{62}$ ».

En 1967, après que l'expansion de la mine eut consommé 54\% du territoire de la ville et engendré la destruction de 250 bâtiments, Asbestos se retrouva dépourvue de centre-ville et ses habitants durent développer un nouveau sentiment d'appartenance au lieu et au milieu ${ }^{63}$. Compte tenu des progrès technologiques réalisés par la Johns-Manville, et malgré la croissance exponentielle des niveaux d'extraction, seuls 200 employés supplémentaires avaient été embauchés à la mine depuis 1949. Le Canadian Mining Journal consacra en 1967 un article au "producteur d'amiante le plus important du monde libre», faisant ainsi la comparaison avec l'essor commercial que connaissait l'Union soviétique. Le journal s'étonnait de la façon dont la Johns-Manville avait transformé la communauté en un «complexe industriel» capable de produire annuellement plus de 600000 tonnes de minerai ${ }^{64}$. Le Canadian Mining Journal oubliait néanmoins de mentionner la réponse locale face à cette transformation.

Si la Johns-Manville était à Asbestos pour les affaires, les travailleurs de la mine Jeffrey y étaient pour vivre. En avril 1968, l'agrandissement de la mine devenait insupportable pour bien des membres de la communauté qui considéraient qu'ils en avaient «soupé de la poussière et du bruit ${ }^{65}$ ". Dans la mesure où la nouvelle machinerie produisait plus de poussière que jamais, Asbestos était devenue un milieu de vie inapproprié pour les familles. Les citoyens demandèrent aux membres du conseil sinon de rediriger les plans d'agrandissement de la Johns-Manville, du moins d'établir une zone tampon de 1000 pieds entre la fosse et la ville ${ }^{66}$. La demande constitua un changement significatif quant à la façon dont la population locale interagissait avec la fosse.

Le conseil mit en place la zone tampon en septembre 1968. Cela permit à la Johns-Manville de démolir davantage de routes et de maisons, et

61. Ville d'Asbestos, Procès-verbal, 4 novembre 1964, p. 7.

62. Le Citoyen, 19 décembre 1964, p. 4.

63. Ross, "Encroachment of the Jeffrey Mine... », loc. cit., 534.

64. "Asbestos", Canadian Mining Journal, 88, 5 (mai 1967): 45.

65. Le Citoyen, 23 avril 1968, p. 1.

66. Ville d'Asbestos, Procès-verbal, 17 avril 1968, p. 62. 
d'offrir $20000 \$$ au conseil municipal pour le développement urbain ${ }^{67}$. La zone tampon fut renforcée en juillet 1969 par la construction d'un mur d'une longueur de 200 pieds à son extrémité. Pour les habitants, ce mur qui leur cachait la fosse en même temps qu'il les protégeait des projections de roche représentait une forme de contrôle sur le territoire et l'espace.

Ce contrôle fut accru en septembre 1969 lorsque le gouvernement du Québec décida que la population d'Asbestos devait approuver la prochaine phase d'agrandissement de la mine ${ }^{68}$. Que l'entreprise n'eut pas reçu carte blanche pour exploiter la terre comme elle le souhaitait était d'autant plus significatif que celle-ci était tout de même à la tête d'une industrie valant, en 1969, 196,8 millions de dollars. L'agrandissement effréné de la fosse devait élever le niveau de production annuelle à 100000 tonnes de fibres ${ }^{69}$, mais à la suite de cette législation, la Johns-Manville se concentra sur l'extraction des fibres des tas de résidus qui faisaient partie du paysage depuis les années 1930. L’agrandissement de la mine était en suspens.

En ralentissant les projets d'agrandissement, l'exploitation des résidus contribua à l'amélioration des relations entreprise-communauté. La ville ne pouvait grandir au même rythme que la mine Jeffrey: même si la croissance de la population était assez faible, les habitants contraints au déplacement par l'agrandissement de la mine n'avaient plus d'endroit où aller. Lorsqu'en 1971, la Johns-Manville expliqua au gouvernement que l'entreprise n'était pas responsable de la pénurie de logement, le conseil municipal riposta en affublant les responsables de l'entreprise du sobriquet de «Ponce Pilate ${ }^{70}$ ». Beaucoup de familles n'avaient d'autre choix que de vivre en une proximité dangereuse avec la mine Jeffrey, mais l'entreprise considérait que tel n'était pas son problème. De plus, certains dirigeants se rendaient compte que les effets nocifs du minerai sur la santé limitaient, de fait, l'avenir de la communauté et de l'industrie. Ainsi, la Johns-Manville commença à porter son attention exclusivement sur le territoire et à se décharger de toute responsabilité envers les habitants d'Asbestos.

\section{CHANGEMENT DE DIRECTION, CHANGEMENT DE CAP, 1972-1983}

Malgré les difficultés auxquelles l'industrie faisait face, le sentiment de confiance qui gagnait les habitants d'Asbestos était d'autant plus fort qu'il animait désormais non plus les seuls commerçants, comme lors des pro-

67. Ville d'Asbestos, Procès-verbal, 2 octobre 1968, p. 117 et 5 mars 1969, p. 180.

68. Ville d'Asbestos, Procès-verbal, 29 septembre 1969, p. 250.

69. "Asbestos", Canadian Mining Journal, 91, 2 (février 1970): 146.

70. Le Citoyen, 22 décembre 1970, p. 1. 
testations contre la première vague d'expropriation, mais l'ensemble de la communauté. Un tiers de la production de la fibre reposait à présent sur les détritus entassés depuis les années 1930, et la Johns-Manville nourrissait l'ambition d'intensifier l'extraction à l'intérieur de la fosse. Alors que l'industrie enregistrait un chiffre d'affaires annuel supérieur à 210 millions de dollars en 1972, l'entreprise n'en visait pas moins une production, pour l'année 1975, de 700000 tonnes $^{71}$. Le tonnage souhaité était d'autant plus aberrant qu'il inonderait le marché d'un minerai que le monde occidental, de plus en plus sensible à ses effets nocifs sur la santé, commençait à rejeter. Avant que l'industrie ne s'effondre, la JohnsManville entendait extraire autant de minerai que possible. Accroître à ce point la production nécessitait un agrandissement si rapide et si exponentiel de la mine Jeffrey qu'à terme, la ville d'Asbestos serait engloutie par la fosse grandissante, tandis que l'équilibre entre l'espace de vie et l'espace de travail serait rompu.

La population rechignait à laisser la Johns-Manville poursuivre ses projets. Décrivant l'évacuation forcée des citoyens de leur maison sur le point de s'engouffrer dans la fosse, le journal local commençait à critiquer la minière $^{72}$, phénomène inexistant dans les autres communautés minières d'amiante du Québec, même à Thetford Mines. Le conseil ne tarda pas à fermer les tronçons routiers jugés dangereux, et la Johns-Manville annonça que 160 résidents devaient déménager afin que la fosse puisse être élargie ${ }^{73}$. Enracinée dans un équilibre étroit entre la population et le territoire, la communauté d'Asbestos ne souhaitait pas compromettre cette coexistence pour satisfaire les seules ambitions de la Johns-Manville. Les 160 habitants refusèrent unanimement de déménager.

À ce stade, la communauté avait conscience qu’elle ne bénéficierait pas des profits engendrés par une extraction accrue. En 1975, dans un contexte d'avancée technologique associé à un ralentissement du marché mondial, la Johns-Manville licencia 154 travailleurs $^{74}$. L'industrie continuait de valoir des millions de dollars, mais au fur et à mesure que changeait l'opinion mondiale au sujet de l'amiante, c'est l'importance de la mine Jeffrey qui se modifiait. L'expansion rapide de la fosse et le refus des familles de quitter les maisons donnaient lieu à de plus en plus d'accidents, dus à l'abattage par explosif et la projection de roches. Les projets d'agrandis-

71. "Asbestos», Canadian Mining Journal, 93, 2 (février 1972): 139.

72. "Mauvaise mine», Le Citoyen, 28 janvier 1975, p. 4.

73. «Mais, pour aller où?», Le Citoyen, 15 avri 1975, p. 3.

74. Le Citoyen, 4 février 1975, p. 3. 
sement affectaient également la mine elle-même. Durant l'année 1975, au sud-est de la fosse, des éboulis de falaise détruisirent d'importantes parties des gradins en spirale. Le conseil municipal demanda au gouvernement provincial de protéger la communauté en contraignant la JohnsManville à limiter ses projets d'agrandissement ${ }^{75}$.

Avec des projections continues de roche, en tout lieu et à toute heure, Asbestos devenait de plus en plus dangereuse. En 1976, des projectiles tombèrent sur l'un des rares magasins encore ouverts, atteignant le fils du propriétaire. Quelques mois plus tard, un éclat de roche traversa le toit de la propriété de M. Hyppolyte, et atterrit dans le couffin, vide, de son bébé $^{76}$. Tandis que le gouvernement somma la Johns-Manville de mettre en œuvre un abattage par explosif plus prudent, l'entreprise choisit plutôt d'investir 77 millions de dollars dans l'acquisition d'un nouvel équipement et d'une nouvelle usine pour accroître les niveaux de production en intensifiant l'abattage par explosif ${ }^{77}$.

Les habitants et la Johns-Manville avaient des vues radicalement opposées quant à la façon dont le territoire devait être occupé. Les premiers considéraient que la communauté comme la mine Jeffrey devaient entretenir une relation équilibrée entre progrès et stabilité. En 1977, le conseil municipal qualifia les éclats de roche d'actes de vandalisme et considéra que les rafales de poussière, toujours plus nombreuses et plus épaisses, avaient rendu insupportable la vie à Asbestos $^{78}$. Des événements similaires se produisirent à Thetford Mines en 1966, où des éclats de roche endommagèrent des habitations et des propriétés privées, et La Tribune de Sherbrooke qualifia la situation de "règne de la terreur ${ }^{79}$ ». Nuages épais de poussière toxique, éclats de roche et bruits de machinerie constants avaient transformé les deux communautés en milieux horribles.

La préoccupation de la Johns-Manville demeura d'extraire de la mine Jeffrey le plus de fibre et le plus rapidement possible. En 1977, un article du Canadian Mining Journal annonça qu'au cours des trois prochaines années, la Johns-Manville aurait détruit plus de 200 immeubles afin de faciliter la croissance de la mine. Le journal considéra ces actions excusables puisqu’à ses yeux, «Asbestos est après tout une assez vieille ville, et...

75. Bibliothèque et Archives nationales du Québec (BAnQ), Fonds du ministère des Ressources naturelles et de la Faune, E78 S999, 7D 024 02-01-001A-01; 1985-02-006 \2, 5 juin 1975.

76. BAnQ, Fonds du ministère des Ressources naturelles et de la Faune, E78 S999, 7D 024 02-01001A-01; 1985-02-006 2, Yves Hamel, Asbestos, à Yves Bérubé 10 octobre 1979.

77. "Asbestos", Canadian Mining Journal, 98, 2 (février 1977): 125.

78. Ville d'Asbestos, Procès-verbal, 18 mai 1977.

79. La Tribune, 3 août 1966, dans F. Cinq-Mars et R Dubé, op. cit., 392. 
[c'est] une forme de rénovation urbaine forcée qui a jusqu'ici profité à Asbestos, une ville assez bien aménagée qui offre à ses habitants les équipements les plus modernes et les plus performants ${ }^{80}$ ».

Avec 6500 pieds d'est en ouest, 6000 pieds du nord au sud et 1000 pieds de profondeur, l'immensité de la mine Jeffrey rendait la vie d'autant plus difficile que la Johns-Manville poursuivait l'industrialisation du territoire et de la population. Les transformations orchestrées à la fin des années 1970 permirent à l'entreprise d'engranger les bénéfices les plus importants de son histoire. Ces transformations nécessitèrent également moins de main-d'œuvre, et 60 employés furent licenciés en 1977. L’année suivante, les travailleurs québécois de l'amiante se mirent de nouveau en grève. Cependant, tandis que les travailleurs de Thetford Mines réclamèrent des mesures anti-poussière supplémentaires, ceux de la mine Jeffrey cantonnèrent leurs exigences à la stabilité de l'emploi et du salaire. En janvier 1978, un dynamitage propulsa trois gros rochers. L'un s'écrasa rue SaintJacques sur la maison d'Adrien Sirois; l'autre, de 23 livres, se fracassa rue Notre-Dame sur la façade de la maison d'Yvette Boucher, à 1800 pieds de la fosse ; et le dernier détruisit la cuisine d'un autre habitant ${ }^{81}$.

Les victimes des dynamitages dénoncèrent la Johns-Manville à la police et au gouvernement. Pour eux, il était question d'agression, et non d'activité industrielle. La province contraignit l'entreprise à prévoir des dynamitages à heures fixes, afin que les habitants puissent évacuer leurs habitations. Bien qu'involontaires, ces accidents permirent à l'entreprise d'exproprier les maisons les plus proches de la mine Jeffrey, et ce, malgré le refus des propriétaires. Marie Fortin-Drouin apprit, en septembre 1979, que sa maison n'était plus sécuritaire ${ }^{82}$. Les employés de la Johns-Manville l'aidèrent à déménager. Une semaine après l'expropriation de la propriété, un éclat de roche traversa le toit du funérarium Desrochers, détruisant une statuette de Jésus. Dans les rues principales, plusieurs voitures et fenêtres furent également endommagées ${ }^{83}$.

Ces incidents permirent à la Johns-Manville d'exproprier en mai 1980 les maisons et les commerces situés à proximité de la mine Jeffrey. Ils

80. Richard Fish, "Canadian Johns-Manville Beginning \$77 million Investment Program», Canadian Mining Journal, 98, 11 (novembre 1977): 8. Nous traduisons.

81. Procès-verbal, Ville d'Asbestos, 18 janvier 1978.

82. BAnQ, Fonds du ministère des Ressources naturelles et de la Faune, E78 S999, 7D 024 02-01001A-01; 1985-02-006 2, Gérard Piché, CJM Administrative Services à Marie Fortin-Drouin, septembre 1979.

83. BAnQ, Fonds du ministère des Ressources naturelles et de la Faune, E78 S999, 7D 024 02-01001A-01 ; 1985-02-006 2, Desrochers à Gouvernement du Québec, septembre 1979. 
suscitèrent également la colère des habitants d'Asbestos, de plus en plus irrités par la façon dont l'entreprise traitait leur communauté et leur territoire. Les habitants cherchèrent d'autres façons de contrôler davantage le territoire d'Asbestos. En septembre 1980, le conseil signifia à la JohnsManville que ses gestionnaires devraient négocier individuellement, avec chaque propriétaire, la vente des terrains ${ }^{84}$.

Au début des années 1980, la prise de conscience grandissante des maladies liées à l'amiante précipita la chute du marché mondial du minerai ${ }^{85}$, et la puissance de la Johns-Manville s'effrita à l'échelle locale et mondiale. Le Québec n'extrayait maintenant que $22 \%$ de l'amiante produite dans le monde, et l'emploi comme les recettes fiscales diminuaient au fur et à mesure que l'Union soviétique dominait le marché ${ }^{86}$. En janvier 1981, exaspérés par le bruit et la poussière en provenance de la fosse, plusieurs habitants s'enquirent auprès du conseil d'une éventuelle loi municipale ou provinciale établissant un périmètre de sécurité entre l'habitat résidentiel et le "cratère d'exploitation» (working crater) qu'était désormais la mine ${ }^{87}$. Mais lorsque le conseil demanda à la Province d'établir une zone tampon d'au moins 150 pieds entre la mine Jeffrey et la communauté, le ministère de l'Énergie et des Ressources répondit qu'une telle mesure était inconcevable, en dépit des plaintes formulées ${ }^{88}$. En 1981, la zone tampon de 1000 pieds qu'avait établie l'entreprise en 1968 avait disparu, et maintenir l'industrie à flot était alors la priorité. Son dynamisme économique avait beau être amoindri, la Johns-Manville demeurait un acteur financier puissant, rapportant à elle seule au Québec 60886000 \$ de recettes fiscales. La Province ne limiterait pas la production. La JohnsManville continua d'acquérir des parcelles individuelles de terre lorsqu'elle annonça, en mai 1981, son intention de réduire ses effectifs de 2200 employés à $1500^{89}$.

La Johns-Manville suscita encore davantage d'angoisse, quand, en mai 1982, elle se plaça sous la protection de la loi sur les faillites aux États-Unis. Selon le journal local, sans la Johns-Manville, Asbestos mourrait ${ }^{90}$. Inquiet de la santé financière de l'entreprise et déterminé à obtenir la garantie

84. Ville d'Asbestos, Procès-verbal, 17 septembre 1980, p. 109.

85. "Asbestos", Canadian Mining Journal, 102, 2 (février 1981): 129.

86. M. Vallières, www.mern.gouv.qc.ca/mines/desminesetdeshommes/index.jsp\#annexe [consulté le 12 janvier 2015].

87. Ville d'Asbestos, Procès-verbal, 21 janvier 1981, p. 171.

88. Ville d'Asbestos, Procès-verbal, 5 mai 1981, p. 222.

89. Ville d'Asbestos, Procès-verbal, 19 mai 1981, p. 235 ; Canadian Mining Journal, 103, 2 (février 1982): 122.

90. Le Citoyen, 10 août 1982, p. 2. 
que les habitants d'Asbestos ne perdraient pas leur maison sans compensation, le conseil municipal demanda pour l'année 1983 la facturation et le paiement relatifs à la vente immédiate des terres. Il était temps pour la communauté de prendre le contrôle de son territoire et de son avenir.

Les habitants devaient désormais mettre dans la balance leurs souvenirs d'un passé glorieux et profitable, de même que leurs craintes concernant l'avenir, puisqu' "il apparaissait très clair que l'âge d'or de l'amiante était terminé et qu'une nouvelle bataille s'annonçait, plus difficile encore, pour la survie de la communauté et de son industrie centenaire ${ }^{91} »$. À cet égard, "usant d'une imagination toujours plus aiguë pour dynamiser et revivifier une communauté qui en a bien besoin, la Ville se démènera avec une énergie insoupçonnée afin de conserver ce qui lui reste: son âme ${ }^{92}$ ».

\section{CONCLUSION}

La Johns-Manville quitta Asbestos en 1983. Elle vendit la mine Jeffrey pour 117 millions de dollars aux 12 entreprises étrangères qui pensaient pouvoir gérer le territoire, la communauté et l'industrie, malgré l'effondrement continu de la demande en amiante ${ }^{93}$. Même si elles ne disposaient pas des réseaux internationaux de la Johns-Manville et du pouvoir qu'ils lui conféraient, les populations locales obtenaient soudainement davantage de contrôle sur leur territoire. Les profits continuaient de s'amenuiser, l'angoisse de croître, et pourtant la mine produisait 30000 tonnes de fibre par jour.

La communauté fit preuve d'optimisme lorsqu'elle déclara 1984, «l’année de l'amiante». Le conseil municipal cherchait à contrecarrer la campagne contre l'amiante menée par l'Environmental Protection Agency du gouvernement américain. C'était à la communauté de restaurer la réputation du minerai, dont la survie de la ville dépendait ${ }^{94}$. Les habitants se souciaient moins de leur santé que de la nécessité de nourrir leur famille. D’ailleurs, la population estimait qu'une mauvaise publicité, et non des preuves médicales, était à l'origine des dégâts que subissait alors l'industrie.

L'histoire de l'occupation et de la transformation du territoire à Asbestos contribue de diverses façons à la littérature relative aux communautés minières. Elle apporte un éclairage sur les conséquences locales des ambitions d'une entreprise multinationale. Ces ambitions, il faut le souligner,

91. R. Lampron et al., op. cit., 335.

92. Ibid.

93. G. O. Vagt, "Asbestos», Canadian Mining Journal, 105, 2 (février 1984): 143.

94. Ville d'Asbestos, Procès-verbal, 19 décembre 1983, p. 143. 
furent nourries pendant des décennies malgré les connaissances des effets néfastes sur la santé de la communauté et de ses membres en contact avec l'amiante. Une grande partie de l'environnement local fut extrait, traité et expédié dans le monde entier. Cependant, le processus de négociation requis pour atteindre de pareils niveaux d'extraction n'était associé ni à un consentement systématique ni même à la présomption d'un consentement.

Ce texte révèle les relations complexes et évolutives que les communautés dépendantes des ressources naturelles entretiennent avec leur environnement local et les entreprises étrangères qui bien souvent le contrôlent. L'histoire relatée ici n'est pas celle d'une multinationale tout simplement libre de transformer le paysage sans avoir obtenu le consentement des populations locales. Chaque fois que la Johns-Manville souhaitait agrandir la mine Jeffrey, la population locale affirmait sa capacité d'action en soutenant ou en contestant le projet, leur choix dépendant avant tout des bienfaits que celui-ci pouvait apporter à la communauté, et non à l'industrie. Si la Johns-Manville comptait occasionnellement sur l'intervention du gouvernement pour mettre en ouvre ses projets d'expansion, c'était avant tout parce que seule la classe des petits commerçants s'y opposait. Pour la majorité des habitants, la transformation massive du paysage d'Asbestos garantissait la pérennité des emplois. Lorsque l'industrie commença à péricliter et que les projets d'extraction de la JohnsManville se firent de plus en plus agressifs, l'emprise de la mine sur le territoire et la communauté prit soudainement une tournure sinistre, mais jamais au point que l'arrêt de l'activité industrielle n'ait été souhaité par les populations. En effet, dès que la minière eut quitté la communauté et que les projets d'extension furent suspendus, les habitants d'Asbestos se mobilisèrent pour sauver les activités de la mine.

Dans le cadre d'une étude fédérale-provinciale sur les villes minières en déclin durant les années 1980, des chercheurs reconnurent le rôle joué par l'environnement local lorsqu'il s'agissait pour les membres de la communauté de comprendre qui - et où - ils étaient ${ }^{95}$. La façon dont les habitants d'Asbestos firent face à l'effondrement de leur industrie révèle combien ils étaient attachés à la mine et au territoire en transformation permanente. Si l'argent constituait certainement un facteur déterminant, il y avait un profond sentiment d'appartenance au lieu, et ce sentiment 
était ancré dans le territoire qui reliait les habitants à la mine Jeffrey. Malgré une histoire d'expropriations répétées, l'absence brutale d'expansion territoriale et économique de la mine Jeffrey fut plus traumatisante que la disparition de l'église ou du centre-ville commercial. La JohnsManville avait fait en sorte que les populations locales perçoivent les transformations territoriales à grande échelle comme le symbole ultime du progrès et de la prospérité. Sans elles, la ville était perdue.

Traduction: Guillaume Blanc 